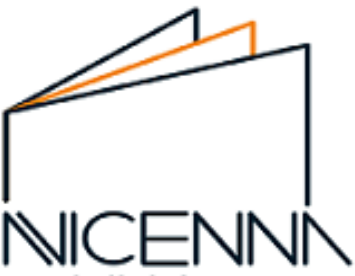

publishing $\mathrm{co}$.

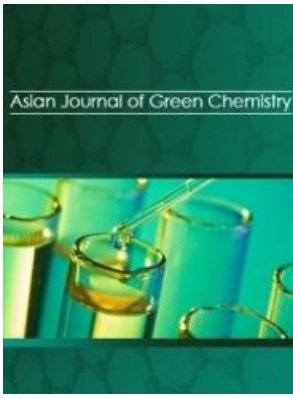

Orginal Research Article

\title{
Synthesis and characterization of nanocomposite based on polymer as a highly effective supported catalyst in oxidation of alkenes
}

\author{
Hossein Salavatia ${ }^{*}$, Abbas Teimouria ${ }^{\text {, Shahnaz Kazemi }}{ }^{\mathrm{b}}$ \\ a Chemistry Department, Payame Noor University, 19395-3697, Tehran, I. R. of Iran \\ b Department of Chemistry, Birjand University, 97179-414, Birjand, Iran
}

\section{ARTICLE INFORMATION}

Received: 6 August 2017

Received in revised: 2 September 2017

Accepted: 2 September 2017

Available online: 24 September 2017

DOI:10.22631/ajgc.2017.94610.1012

\section{KEYWORDS}

\section{Heteropolyacid}

Polymer

Oxidation

Alkenes

Support

\section{ABSTRACT}

Polyphosphotungstate (denoted as PPT) was supported on polypyrrol as organic support (abbreviated as PTT@Ppy) to produce catalytic active supported catalyst. This catalyst was characterized using X-ray diffraction (XRD), Fourier-transform infrared spectroscopy (FT-IR), Field emission scanning electron microscopy (FESEM) and UV-vis diffuse reflectance spectroscopy (UV-DRS). The catalyst showed high catalytic activity in the oxidation of alkenes under optimized conditions. In this work, cyclooctene was selected as model alkene for investigation of oxidation procedure, and then under optimized condition, other alkenes were examined. The catalyst could be readily separated from the catalytic system using the centrifuging and loss of activity was negligible when the catalyst was recovered in five consecutive cycles. For this research, a statistical method called response surface methodology (RSM) has been used to economize the number of experiments and their meaningful interpretation. The effect of various factors such as catalyst amount, time, oxidant amount and temperature on oxidation of alkenes were investigated. Optimization results for $0.2 \mathrm{mmol}$ cyclooctene showed that maximum oxidation efficiency $88 \%$ was achieved at the optimum conditions: catalyst amount $200 \mathrm{mg}$, temperature 63 , time 5 $\mathrm{h}$ and oxidant $=2.15 \mathrm{mmol}$.

\section{Graphical Abstract}

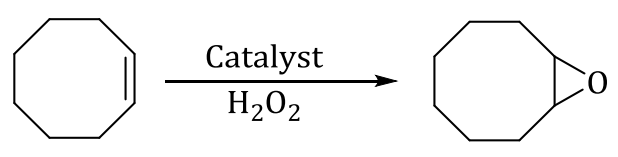




\section{Introduction}

Catalysis by heteropoly acids (HPAs) and related polyoxometalate compounds is a field of increasing importance. HPAs have several advantages as catalysts which make them economically and environmentally attractive [1-3]. On the other hand, HPAs have a very strong Brønsted acidity, approaching the super acid region on the other, they are efficient oxidants, exhibiting fast reversible multi electron redox transformations under rather mild conditions. Their acid-base and redox properties can be varied over a wide range by changing the chemical composition [4]. The most important advantages of HPAs are different multi factorial, simple preparation, and change capability of chemical and physical properties. In addition, among various structures of polyoxometalates as participate in catalysts, keggin type hetropolyacid is of particular attention because of its high chemical and mechanical resistance under reaction conditions; also, the lowest vacant d molecular orbital in octahedra $\left(\mathrm{MO}_{6}\right)$ of this structure is a nonbonding metal-centered causing its reversible reduction to form multivalance species [5]. Usually tungsten heteropolyacidic are more in attention because they have high acidic power and less oxidation potential than molybdenum acids. In the last two decades, the broad utility of HPA acid and oxidation catalysis has been demonstrated in a wide variety of synthetically useful selective transformations of organic substances [6]. Several new industrial processes are based on HPA catalysis, such as oxidation of olefins that among these olefins cyclooctene are often used as model compounds to evaluate the possible use of a catalyst in alkene oxidation. Several polyoxometalates have already been used in the oxidation of cyclooctene with various oxidants.

Previous studies on the oxidation of cyclooctene by $\mathrm{H}_{2} \mathrm{O}_{2}$ in $\mathrm{CH}_{3} \mathrm{CN}$ used the sandwich type keggin polyoxometalate [7, 8]. Salavati et al investigated the catalytic activity of plyaniline-based keggin polyoxometalate nanocomposite for oxidation of alkenes [9]. Farsani et al. described the oxidation of cyclooctene with $\mathrm{H}_{2} \mathrm{O}_{2}$ in acetonitrile in the presence of substituted keggin polyoxometalate tungstophosphates [10]. Salavati et al reported on the catalytic activity for epoxidation of alkenes using supported heteropolymolybdate nanoparticles on the Bentonite [11]. There are many studies on polymer nanocomposites and their synthetic methods including many studies in which nanocomposites are involved to find new advanced materials with improved mechanical properties and other researches about electrical, optical, and catalytic properties of the nanocomposites. One of these organic supports is polypyrrole (PPy) which is a type of organic polymer formed by the polymerization of pyrrole. Polypyrroles are conducting polymers, related members being polythiophene, polyaniline, and polyacetylene. In addition, PPy and related conductive polymers have two main applications in electronic devices and for chemical sensors. 
There are many studies on polypyrrol nanocomposites and their application in various fields [12]. Cabera et al investigated electrochemical behaviors of polypyrrol magnetic nanocomposite [13]. Bonaster et al [14] applied polypyrrol/polyoxometal in corrosion of carbon electrodes. Yang et al prepared polymer films of polypyrrol/polyoxometalate [15].

In this study, we applied central composite design (CCD) methods for investigation of combined effects in oxidation of alkenes. Formal batch adsorption studies depend on different process parameters, such as temperature, time, adsorbent dose, oxidant/substrate ratio etc. which are constant at unspecified levels. But, this approach does not determine the combined effect of all the process parameters. For scale-up studies, conventional batch process is time consuming and requires a large number of experiments to determine the optimum levels (which may be unreliable) and thereby, increases the overall cost of the process. The application of statistical methods for optimizing adsorption process involves less treatment time, low costs and higher percentage yields. The mutual interactions between variables are described by the graphical representation of the equations called response surfaces. Design-Expert 7.0.0 software (Stat Ease, USA) was used for this study. In this study, central composite design (CCD) was employed with 30 experiments, including six replicates using RSM. Artificial neural network (ANN) was used for designing the process modeling M [16].

Thereupon, the main aim of the present study is to consider a novel system for catalytic properties using keggin polyoxometal, immobilized over polypyrrol as organic support. The advantage of this catalyst is that we combine the unique properties of the polypyrrol as a carrier for immobilization of catalytic active metal ions as heterogenous catalyst with polyoxometal due to have vacant $\mathrm{d}$ orbitals.

In addition, we describe the catalytic activity of the catalyst in oxidation of alkenes by various oxidants using a synthesized catalyst with two components, polypyrrol as organic conducting polymer support and polyoxometal as owing to have vacant $d$ orbitals for exciting the electron for accelerate electron transfer reactions. The results showed that this catalyst have recovery capability and high reuse and it can be used in reactions several times without being reduced dramatically in their actions.

\section{Experimental}

Matreials and methods

Solvents, reagents and chemicals were obtained from Merck, Fluka or Aldrich chemical companies. The samples were ground into a fine powder for characterization. FT-IR spectra were 
obtained as potassium bromide pellets in the range of $400-4000 \mathrm{~cm}^{-1}$ with a Nicollet-Impact 400D instrument. Scanning electron microscopy (SEM) of the catalysts and supports were taken on SEM Philips XL 30 instrument. Diffuse reflectant spectroscopy (DRS) UV was recorded on a 160 Shimadzu spectrophotometer. X-ray diffraction (XRD) patterns were recorded with a Philips X-ray diffractometer (Model PW1840). The reaction products were identified by GC-MS, based on the mass spectra interpretation and by comparison with the library of the equipment.

Preparation of polypyrrol (abbreviated as Ppy)

Polypyrrol (denoted as Ppy) was synthesized by beforehand reported techniques; to describe it briefly: $0.4 \mathrm{~mL}$ of pyrrol was dissolved in $10 \mathrm{~mL} \mathrm{HCl}(0.01 \mathrm{~N})$ after that $2.88 \mathrm{~g}$ of sodium dodecyl sulfate $\left(\mathrm{C}_{12} \mathrm{H}_{25} \mathrm{NaO}_{4} \mathrm{~S}\right)$ was added to the above solution. Then, the mixture was stirred for $2 \mathrm{~h}$. After that, a solution containing $3.08 \mathrm{~g}$ of $\mathrm{FeCl}_{3} \cdot 6 \mathrm{H}_{2} \mathrm{O}$ was included into the above solution, keeping the temperature at $25{ }^{\circ} \mathrm{C}$ for $30 \mathrm{~min}$. The mixture was centrifuged and the product was washed three times with water and then dried at $80^{\circ} \mathrm{C}$ for $3 \mathrm{~h}$ and $60^{\circ} \mathrm{C}$ for $2 \mathrm{~h}$ [17]. The scheme of polypyrrol was shown in Scheme 1.

Preparation of supported catalyst (denoted as PPT@Ppy)

The polyoxometal supported on polypyrrol was synthesized through electrostatic interaction between positive charge of polypyrrol and negative charge of oxyanions of keggin polyoxometal. The synthesis of HPW supported on polypyrrol was carried out by dissolving HPW (3 g) in DI water $(150 \mathrm{~mL})$. This solution was added slowly to a synthesized polypyrrol with dispersion by sonication for $1 \mathrm{~h}$ at $25{ }^{\circ} \mathrm{C}$. The mixture was stirred to obtain HPW@Ppy nanocomposite. The catalyst was collected using centrifuged and dried under air for $24 \mathrm{~h}$ at $25^{\circ} \mathrm{C}$.

\section{Results and discussion}

\section{Characterization of HPW@Ppy}

The FT-IR spectra of HPW and HPW@Ppy are shown in Figure 1. All samples showed two intense and broad peaks at around 3448 and $1622 \mathrm{~cm}^{-1}$, which were associated with the stretching vibrations of free or adsorbed water remaining in the sample. Characteristic infrared bands in the 780-1190 $\mathrm{cm}^{-1}$ region was observed that could be assigned to keggin structure of HPW. FT-IR spectrum related to catalyst $\mathrm{H}_{3} \mathrm{PW}_{12} \mathrm{O}_{40}$ and $\mathrm{PPy}$ and composite $\mathrm{H}_{3} \mathrm{PW}_{12} \mathrm{O}_{40} / \mathrm{PPy}$ is provided respectively in the Figure 1. Absorptions are seen in $\mathrm{H}_{3} \mathrm{PW}_{12} \mathrm{O}_{40}$ spectrum in the points, 840, 895, 980 and $1076.5 \mathrm{~cm}^{-1}$ respectively related to stretching vibrations of $\mathrm{W}-\mathrm{Oe}-\mathrm{W}, \mathrm{W}=\mathrm{O}, \mathrm{W}-\mathrm{O}_{\mathrm{c}}-\mathrm{W}, \mathrm{P}-\mathrm{O}$ 
(Figure 1a) [18]. In supported catalyst, the peaks were attributed to $1037.52 \mathrm{~cm}^{-1}(\mathrm{P}=0 \mathrm{a}), 960 \mathrm{~cm}^{-1}$ $\left(\mathrm{W}-\mathrm{O}_{\mathrm{b}}-\mathrm{W}\right), 878 \mathrm{~cm}^{-1}(\mathrm{~W}=\mathrm{Ot})$, and $835\left(\mathrm{~W}-\mathrm{O}_{\mathrm{c}}-\mathrm{W}\right)$, respectively (Ot= terminal oxygen, $\mathrm{O}_{\mathrm{b}}=$ bridged oxygen of two octahedral sharing a corner and $\mathrm{O}_{\mathrm{c}}=$ bridged oxygen sharing an edge) were observed and the shift due to the immobilization of keggin polyoxometal on polypyrrol as organic support was occured; this indicated that HPW was supported on polypyrrol .The presence of $\mathrm{C}-\mathrm{N}$ and $\mathrm{N}-\mathrm{H}$ bonds of polypyrrol was confirmed by the peaks at 1467 and $1043 \mathrm{~cm}^{-1}$ (Figure 1b), which was shifted to $1387 \mathrm{~cm}^{-1}, 1037 \mathrm{~cm}^{-1}$ after supporting polyoxometal (Figure 1c).

The electronic properties of HPW@pollypyrrol was studied using DR UV-vis spectroscopy. The DR UV-vis spectra of HPW have absorption bands in the range 300-360 nm [18], the absorption bands were appeared in the DRS spectra of catalysts with shift due to immobilization of HPW on the surface of polypyrrol and, in the catalyst, the peaks in the range 300-360 nm could be subjected to keggin polyoxometal (Figure 2). Therefore, these results showed that primary keggin structure has been immobilized on the surface of polypyrrol and showed strong interaction with catalyst. The broadness of peak related to supporting HPW on the surface of polypyrrol as organic support.

Figure 3 shows the XRD patterns of polypyrrol, HPW and HPW@Ppy. XRD pattern confirmed the primarily amorphous character of PPy. Characteristic peaks of HPW in the keggin structure (Figure 3a) confirmed that HPW was successfully synthesized [18]. The powder XRD pattern $(0<\theta<70)$ of polypyrrol obtained from the samples is shown in Figure $\mathbf{3 b}$ and showed amorphous structure of polymer. Compared with previously reported XRD pattern of PPy, the XRD pattern of the PPy showed similarly structural order. It is observed that the broad peaks of the polymer centered at about 20 is assigned to amorphous structure of polymer, which was close to the distance of polymer repeat unit with relatively distinct bragg reflections. In addition, immobilization of HPW on polypyrrol led to change in the powder XRD pattern when compared with HPW confirm the formation of a nanocomposite material and a possible change in crystallinity and interplanar space. These patterns indicated that HPW was immobilized on the surface position of polypyrrol. In addition, XRD patterns show absence of any impurities in prepared sample. D parameter, which is defined as crystallite size of the catalyst sample, was calculated according to Scherer's equation:

$$
\mathrm{D}=0.9 \lambda / \beta \operatorname{Cos} \theta
$$

Scheme 1. Structure of synthesized polypyrrol

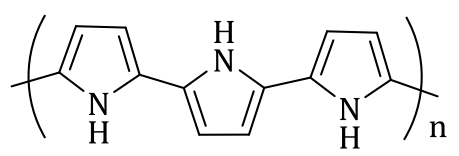


In the mentioned formula, $\lambda$ and $\beta$ refer to the wavelength of $\mathrm{X}$-ray and full width at half maximum of the peak at diffracting angle $\theta$, respectively. For the supported catalyst, the average diameter of particles was estimated to be $35 \mathrm{~nm}$ by averaging its diameters when they are measured in different directions in the FESEM images (Figure 4).

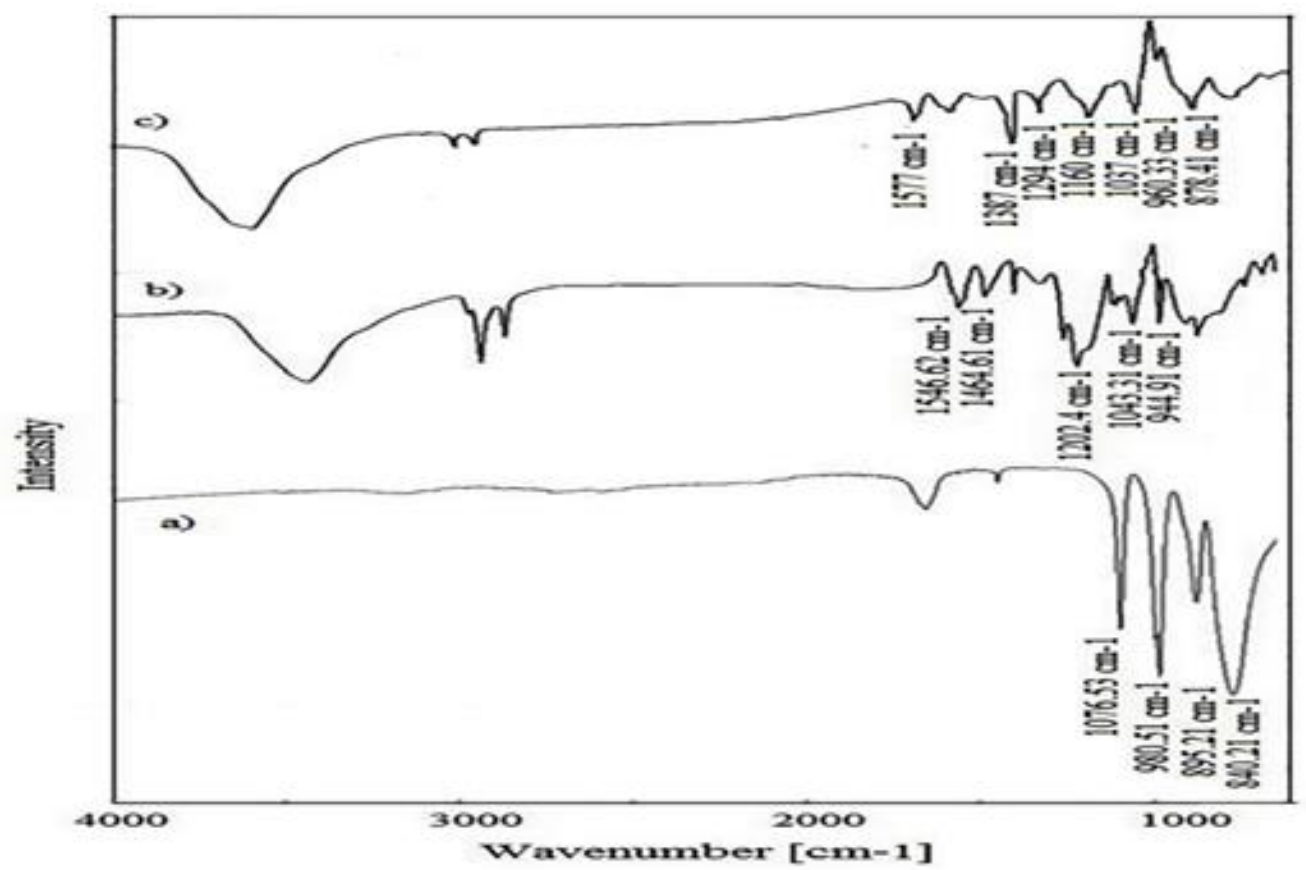

Figure 1. FT-IR spectrum of a) HPW, b) Ppy, c) HPW@Ppy

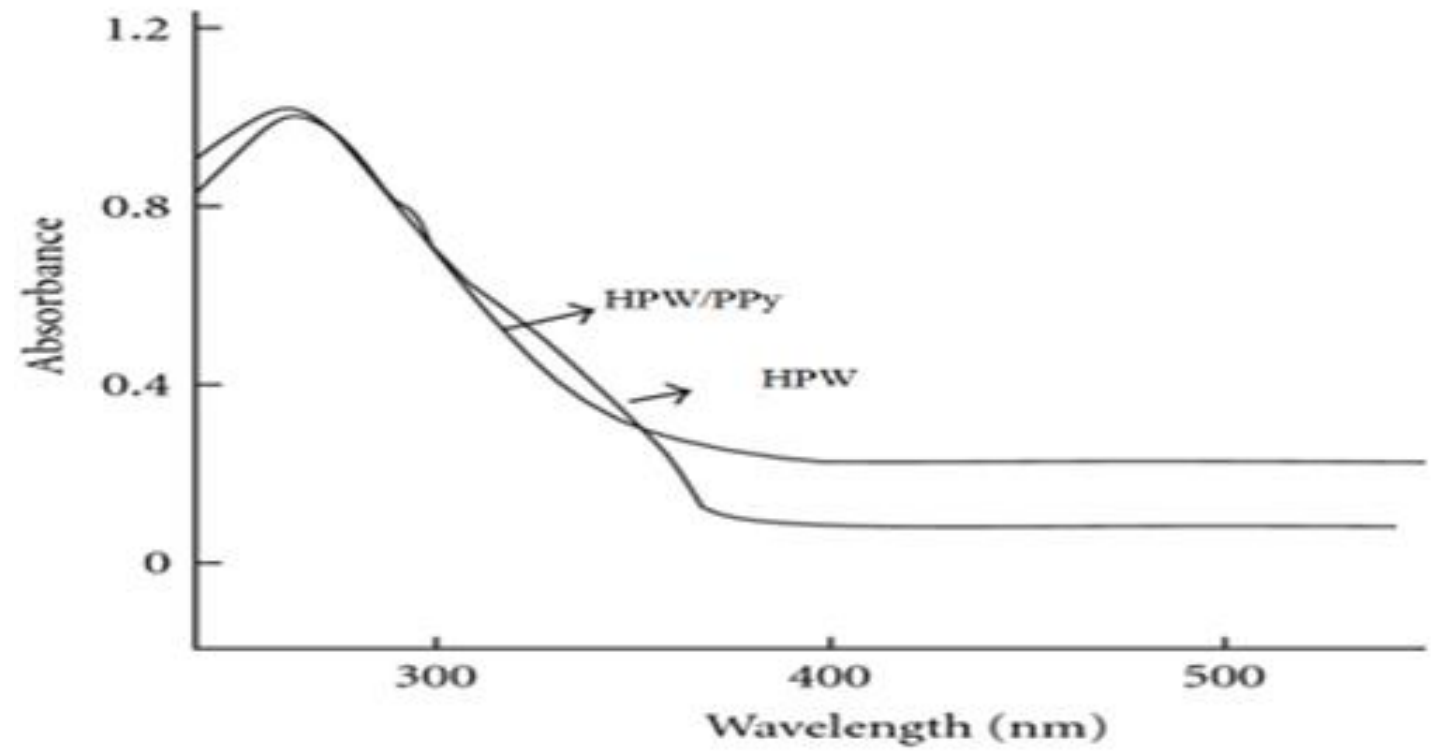

Figure 2. DR-UV spectrum of supported catalyst 
Figure 3. XRD

patterns of a) HPW,

b) Ppy, c) HPW@Ppy

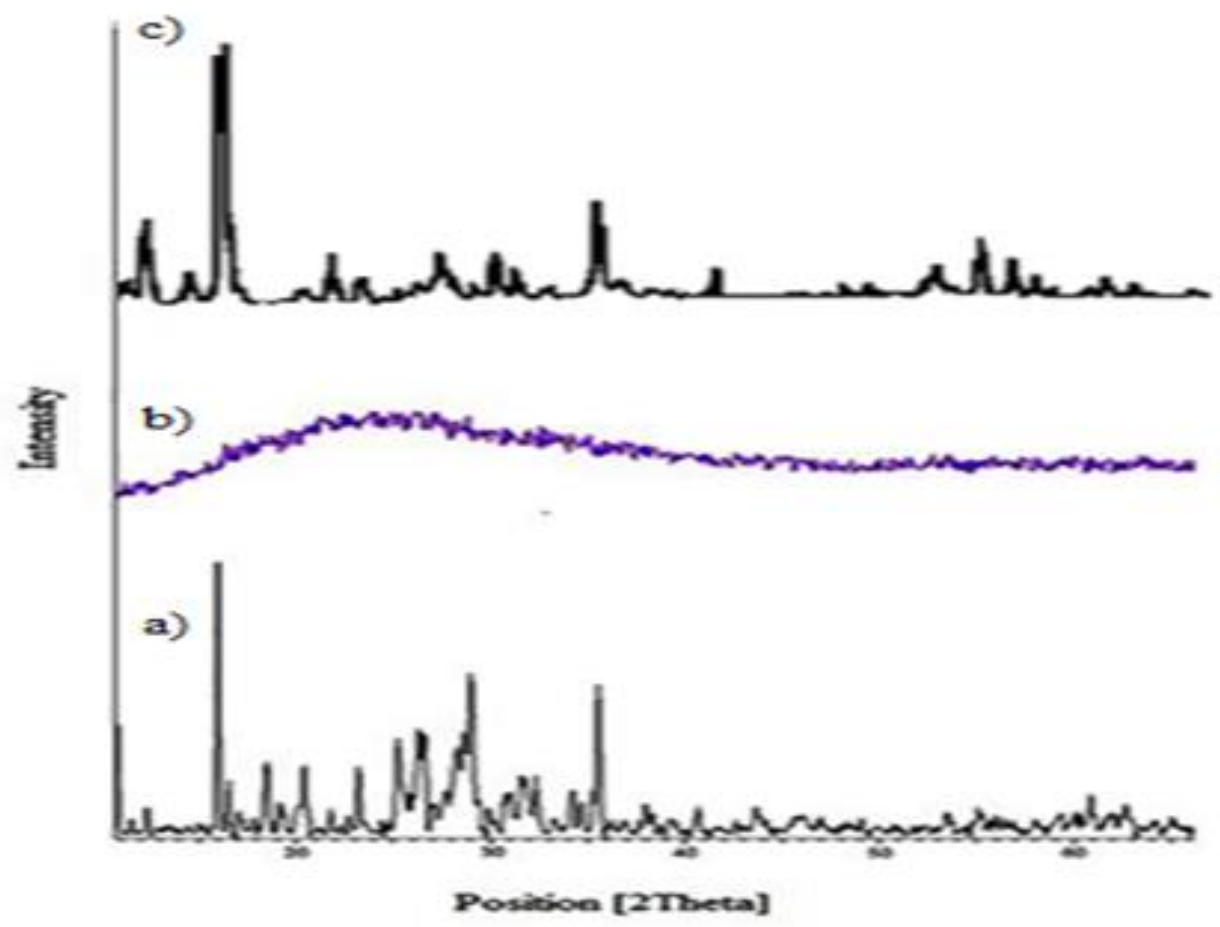

Figure 4. FESEM

image of HPW@Ppy nanocomposite

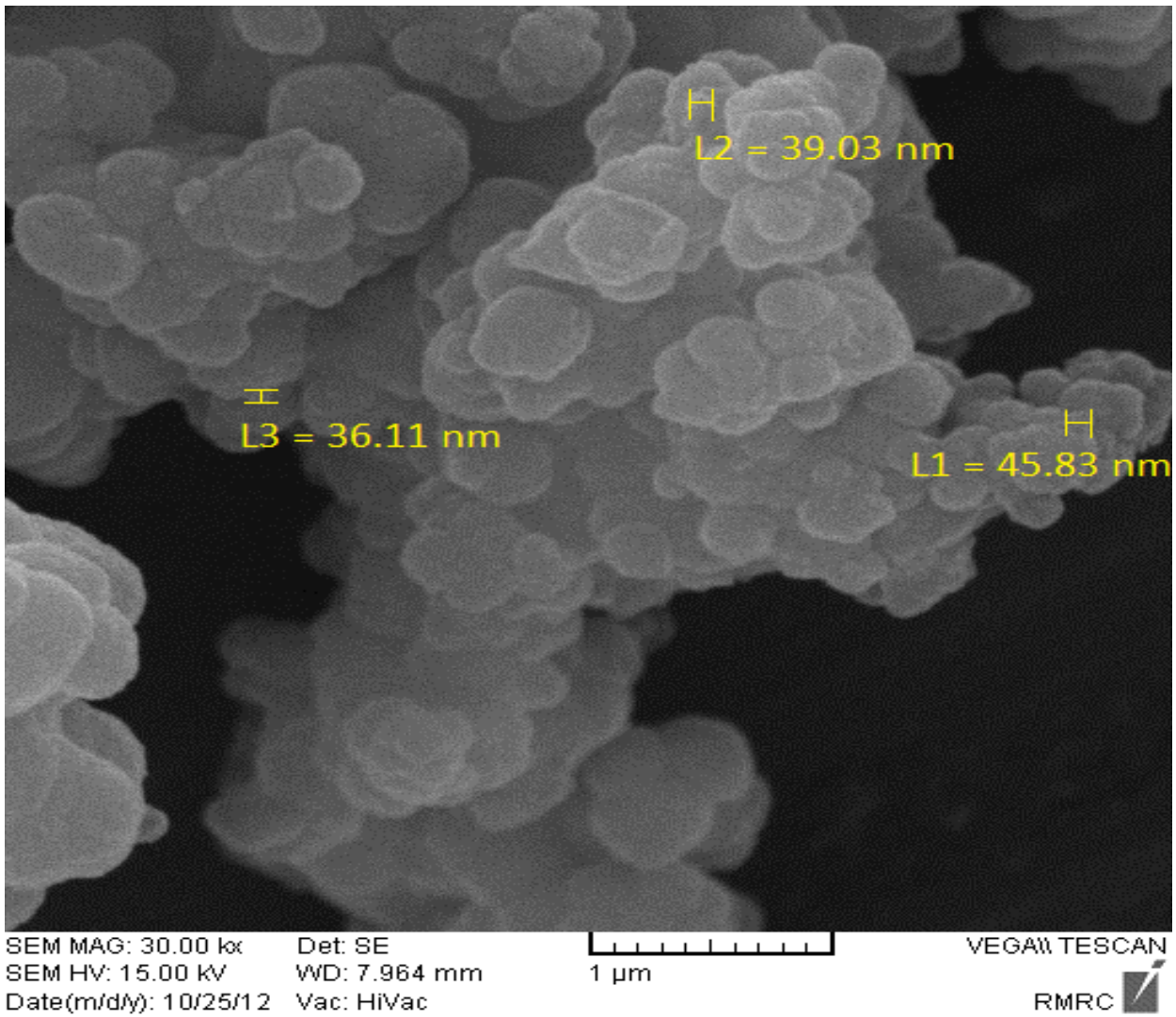


The morphology of HPW and HPW@Ppy supported catalyst was examined using FESEM. Figure 4 show the morphology of supported catalyst. For the supported catalyst, the obtained particles were agglomerated as a consequence of high particle surface energy. The morphologies and nanostructures of the nanoparticles were approximately spherical. Some nanoparticles were importantly aggregated. By assuming that a nanoparticle is spherical, the average diameter of nanoparticles was estimated to be $36-47 \mathrm{~nm}$ ranges. By averaging its diameters measured in several directions in the FESEM images. The larger size could be due to the aggregation of particles. The larger particle size of supported catalyst compare to HPW nanoparticles could be due to immobilization of keggin polyoxometal on the surface of polypyrrol. In addition the texture of organic polymer chain is made of complex polypyrrol, XRD spectrum of this compound and its composite appearance in amorphous.

\section{Oxidation reactions with hydrogen peroxide}

The reactions were typically performed in air at $63^{\circ} \mathrm{C}$, in a stirred solution of the substrate (sub), catalyst (cat) and 30\% (w/w) aqueous hydrogen peroxide (ox) in acetonitrile/THF. The oxidation of alkenes was always carried out in various conditions and the optimized conditions were obtained. The amounts of the several components used in the reactions were dependent on the substrate and are presented in the results and discussion section. The conversion and percentages of each compound in the reaction mixtures were analyzed using gas chromatography. The cyclooctene was selected as model substrate and other alkenes such as limonene, 1-octen, 2-decene, indene and some other alkenes were examined under optimized conditions and the results showed good conversion for examined alkenes. Gas chromatography was carried out under the mentioned condition: for cyclooctene: initial temperature: $80{ }^{\circ} \mathrm{C}(2 \mathrm{~min})$; temperature rate: $20{ }^{\circ} \mathrm{C} / \mathrm{min}$; final temperature: $200^{\circ} \mathrm{C}(2 \mathrm{~min})$; injector temperature: $220^{\circ} \mathrm{C}$; detector temperature: $220^{\circ} \mathrm{C}$.

Effect of various factors on the catalytic oxidation of cyclooctene

The influence of various factors on the catalytic oxidation of alkenes was investigated and we examined effects of temperature, amount of catalyst, and oxidant amount and time on the catalytic oxidation of cyclooctene selected as model alkenes for optimization. The design expert 7.0.0 software was used for regression analysis of the data obtained and to estimate the coefficient of the regression equation. The equations were validated by the statistical tests called the ANOVA, to determine the significance of each term in the equations fitted and to estimate the goodness of fit in each case. Response surfaces were drawn for the experimental results, obtained from the effect of different variables on the percentage removal of dye, in order to determine the individual and 
cumulative effects of these variables and the mutual interactions between them. The Fisher's $\mathrm{F}$ value with a low probability $(\mathrm{p}<0.0001)$ showed that the model was significant. The multiple correlation coefficient $\left(\mathrm{R}^{2}\right)$ demonstrated the goodness of the model. Moreover, $\mathrm{R}^{2}$ value is 0.964 which could be explained by the developed quadratic model, and the predicted $\mathrm{R}^{2}$ values were in agreement with adjusted $\mathrm{R}^{2}$, which means all the terms depicted in the model were significant. In this case, the non-significant lack-of-fit (0.08) confirmed the quadratic model was valid for this process.

Comparison between theoretical and experimental data

The CCD equation of actual factors was solved by partial differential calculus for obtaining the optimum value of $\mathrm{A}, \mathrm{B}, \mathrm{C}$. The optimized value is shown below and Table 1 shows optimized data was obtained for this study. A (time) $=5 \mathrm{~h}, \mathrm{~B}$ (temperature) $=63$, C (oxidant) $=2.15 \mathrm{mmol}$, for catalyst amount $=200 \mathrm{mg}$. The conversion obtained based on optimized condition is shown in Table 1 .

The regression plot of the trained network was shown in Figure 5. The trained network gave a correlation coefficient of 0.964 . A high correlation coefficient of this plot signified the reliability of the neural model with the experimental data.

The model summary statistics results were shown in Table 2. The predicted R-squared of 0.912 is as close to the adjusted R-squared of 0.9334 . This confirmed that your model is acceptable.

The effect of different levels of oxidant amount and time on oxidation of cyclooctene using the keggin polyoxometal supported on polypyrrol can be predicted from the contour plot as shown in Figure 6. From the contour plot, it can be observed that oxidation percentage increased with oxidant amount and time. This could be due to the increase in the exposed surface area of the catalyst. However, it must be noted that after a certain limit, if the amount of oxidant had been increased further, there would have been a saturation point and the conversion was decreased because the oxidant probably decomposed. From the results, it was observed that a maximal removal efficiency of $88 \%$ was achieved at $2.15 \mathrm{mmol}$ oxidant, and $5 \mathrm{~h}$ of time. The combined effect of temperature and time on oxidation of cyclooctene was shown in the contour plot of Figure 6 . The percentage of oxidation was increased by increasing time due to increase in the number of molecules of alkene which converted to epoxide. Higher temperature enhanced the catalytic active sites force and increased the oxidation of alkene. From the results, it was observed that a maximal removal efficiency of $88 \%$ was achieved at $63{ }^{\circ} \mathrm{C}$, and $5 \mathrm{~h}$ of time. The combined effect of temperature and oxidant amount on oxidation of cyclooctene were observed in the contour plot of 
Figure 6. It was shown that $88 \%$ conversion increased with increasing oxidant and temperature. The maximum conversion of $88 \%$ was obtained from catalyst amount $2.15 \mathrm{mmol}$ oxidant and temperature $63.7^{\circ} \mathrm{C}$.

Table 1. Constraints applied for optimization process for oxidation of cyclooctene (catalyst amount $=200 \mathrm{mg}$ )
Temperature $\left({ }^{\circ} \mathrm{C}\right)$ Time (h) Oxidant (mmol)
Epoxide \%

63 5 2.15 $88 \%$

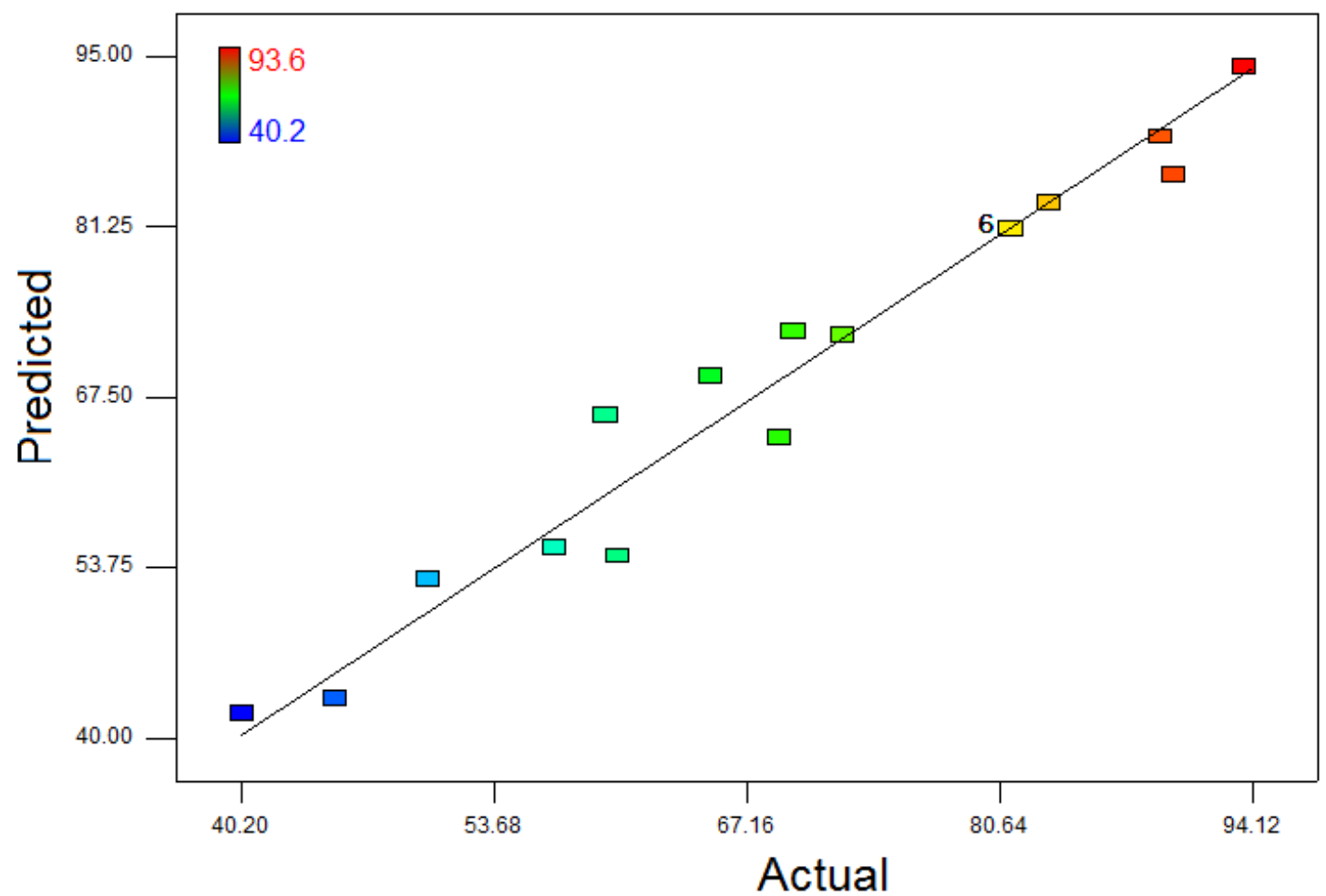

Figure 5. Regression plot (experimentally vs. predicted) using three input variables

Table 2. Model summary statistics

\begin{tabular}{ccccc} 
Source & R-squared & Adjusted R-squared & \multicolumn{2}{c}{ Predicted R-squared } \\
Quadratic & 0.9649 & 0.9334 & 0.9121 & Suggested \\
\hline
\end{tabular}



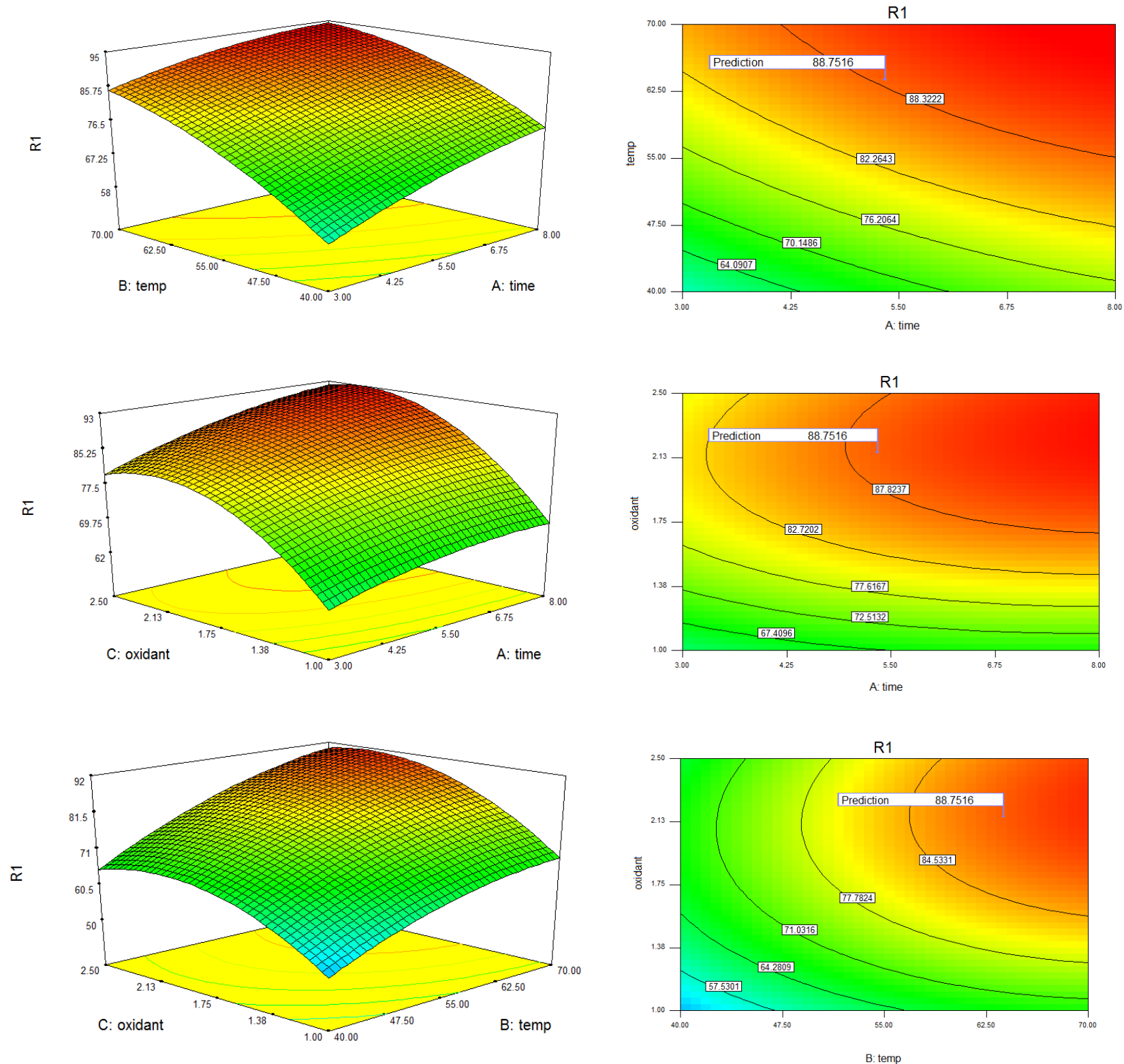

Figure 6. Three dimensional response surface graph and counter plots for the degradation of dye wastewater, \% dye degradation versus time and temperature, counter plots for time and temperature, \% dye degradation versus time and oxidant, counter plots for time and oxidant, \% dye degradation versus temp and oxidant, counter plots for time and oxidant, respectively with $200 \mathrm{mg}$ catalyst.

It should be noted that, when the catalyst was replaced by polypyrrol as catalyst, no oxidation product was observed with longer reaction times up to $24 \mathrm{~h}$ (Figure 7). 


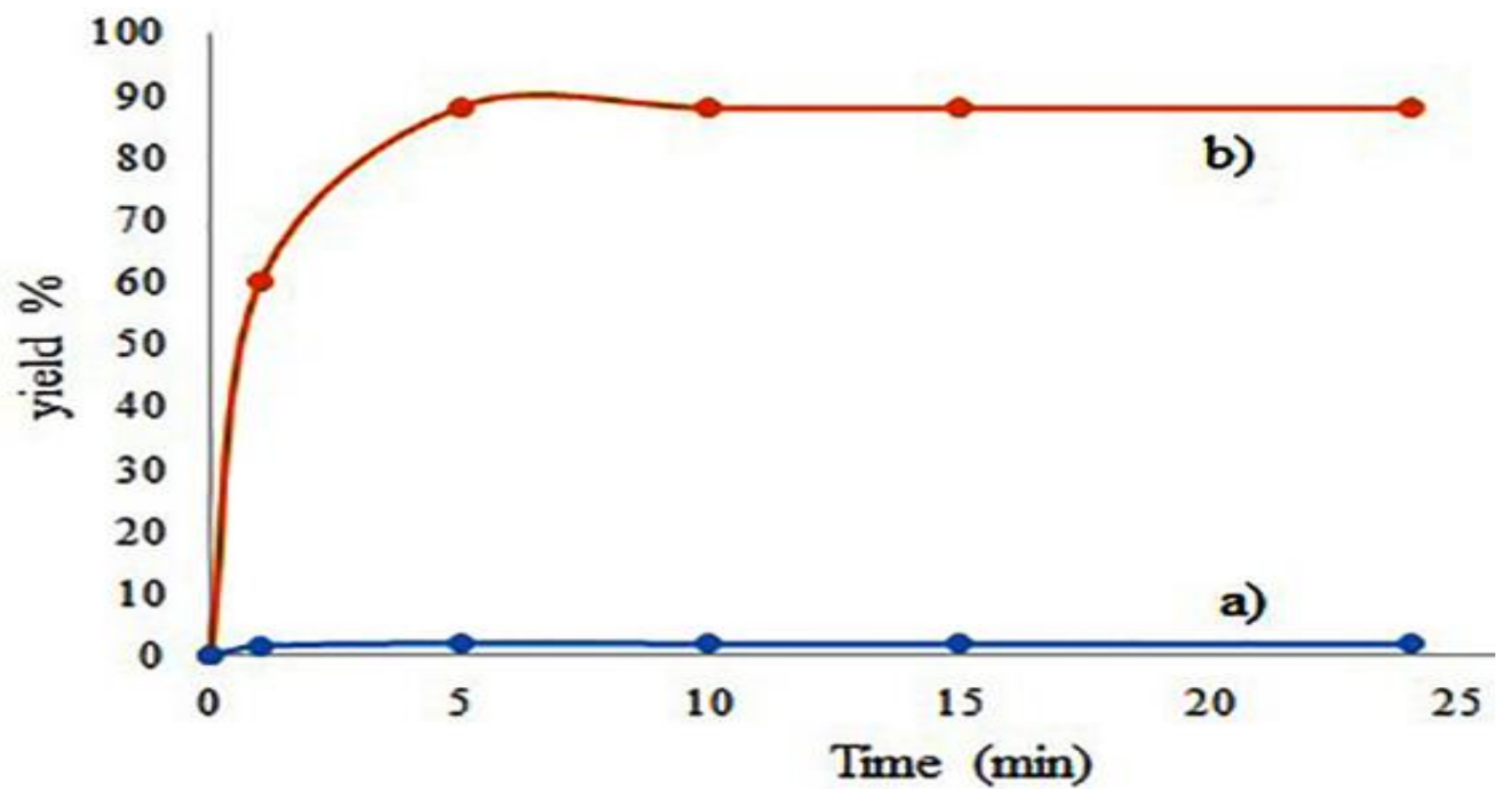

Figure 7. Oxidation of cyclooctene in the presence of a) Polypyrrol as catalyst, b) supported catalyst

To establish the general applicability of the process, various alkenes were subjected to the oxidation system using the synthesized catalyst (Table 3, entries 1-5). The relativities of the alkene compounds were influenced by two main factors, i.e., the electron density on the olefin band and the steric hindrance of the alkene compound.

To investigate the effect of oxidant nature on the conversion of cyclooctene, a series of experiments with various oxidants was applied under the oxidation reaction. The results shown in Figure 8 indicate that the conversion of cyclooctene in the presence of different oxidant was changed from $25 \%$ to $88 \%$. Sufficient catalytic sites were available to drive the reaction to completion in the presence of $\mathrm{H}_{2} \mathrm{O}_{2}$ as oxidant, and was chosen for further experiments. The highest conversion of cyclooctene in the presence of $\mathrm{H}_{2} \mathrm{O}_{2}$ could be due to higher yield of superoxo in the oxidation reaction. In addition, blank reactions with $\mathrm{H}_{2} \mathrm{O}_{2}$ but without catalyst and with catalyst but without $\mathrm{H}_{2} \mathrm{O}_{2}$ were studied. Conversion values attained in the blank reaction were in the range of 3 $7 \%$.

Consideration of acid catalyst properties of synthesized catalyst

The density and strength of acid sites and the rate of catalytic reactions on metal salts catalysts are increased with the slight oxidation of the M centers, which often occurs during catalytic reactions in oxidation media environments. In addition, the more effective dispersion of metal salt species on high surface area supports the introduction of a heteroatom in order to create a 
permanent charge imbalance in the metal oxide structure, thereby increasing the density and strength of the acid sites. According to the definitions offered by BrØnsted and Lewis acidity, a solid acid shows a tendency to donate a proton or accepts an electron pair, respectively. A solid acid can also be defined as a solid on which the color of a basic indicator changes, or as a solid on which a base is chemically adsorbed [19-22]. Thus, the acidic properties of the catalysts should be considered. The proposed mechanism for acid catalyst properties of supported catalyst is shown below; immobilized ion metal in polyoxometalate reacted with $\mathrm{H}_{2} \mathrm{O}_{2}$ to generate peroxo species. This species facilitated the transfer of the electrophilic oxygen to the alkene, yielding the corresponding epoxide. The epoxide product could be easily removed by extraction. Many investigators have suggested various mechanisms for the oxidation of alkenes. Scheme 3 suggests the catalytic oxidation pathways.

Table 3. Oxidation of alkenes to epoxide with $\mathrm{H}_{2} \mathrm{O}_{2}$ catalyzed by synthesized catalyst. GC yields based on the toluene as the internal standard

\begin{tabular}{|c|c|c|c|c|}
\hline Entry & Alkenes & Product & Yield \% & Time (h) \\
\hline 1 & & Epoxide & 88 & 5 \\
\hline 2 & & Epoxide & 85 & 6.30 \\
\hline \multicolumn{5}{|l|}{3} \\
\hline & & Epoxide & 86 & 6 \\
\hline 4 & & Epoxide & 64.2 & 6.30 \\
\hline 5 & & Epoxide & 87 & 7 \\
\hline
\end{tabular}


6

7<smiles>CC1=CCCCC1</smiles>

8<smiles>C=CC1=CCCCC1</smiles>

9
Epoxide

Epoxide

72.3

79.2

Aldehyde

Epoxide

42
6

7

7

7.30

The reactions were run at $63{ }^{\circ} \mathrm{C}$ in $2 \mathrm{~mL}$ of an aqueous suspension of olefin $(0.2 \mathrm{mmol})$, containing $0.2 \mathrm{~g}$ of supported polyoxometal as catalyst with $\mathrm{H}_{2} \mathrm{O}_{2}$ as oxidant. All the reactions were run at least in triplicate, and the yield represent an average of these reactions. The selectivities of epoxides were $>99 \%$. Yields determined by GC.

Scheme 2. Proposed mechanism for oxidation of alkene with the supported catalyst as acid catalyst

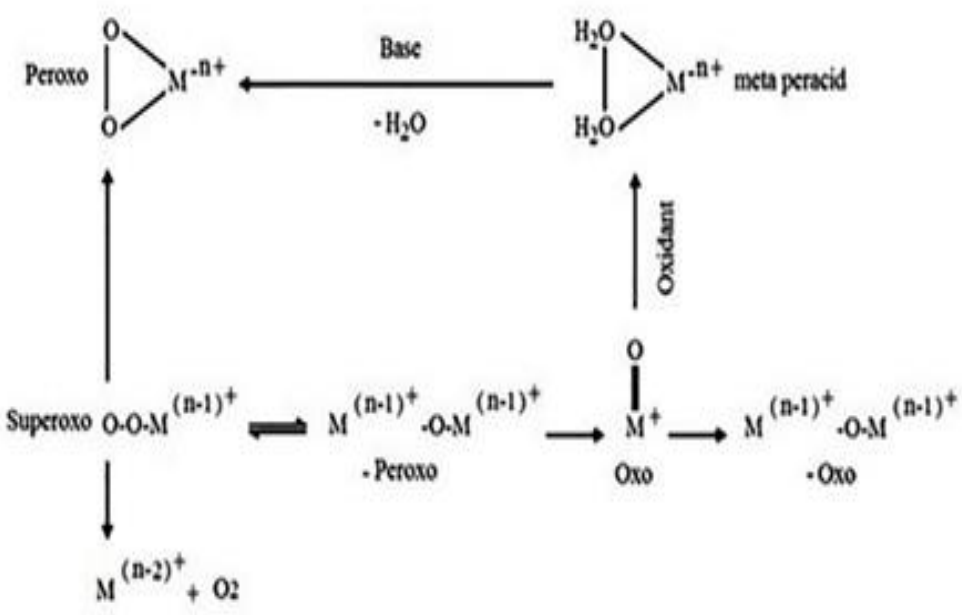

Catalytic behavior, separation, and recyclability

The stability of the supported catalyst was monitored using multiple sequential oxidation of cyclooctene with synthesized catalyst under optimized conditions. For each of the repeated reactions, the catalyst was recovered, washed thoroughly with water to remove the $\mathrm{H}_{2} \mathrm{O}_{2}$ and dried 
before being used with fresh cyclooctene solution. Figure 9 shows that the catalysts were consecutively reused five times. The catalyst was recovered for five runs without the loss of activity.

The assessment of the stability of catalysts was performed as follows: a drop of the reaction mixture was dried on a $\mathrm{KBr}$ pellet and the infrared spectrum measured was taken and the result showed the peaks corresponding to HPW@polypyrrol shown at $870 \mathrm{~cm}^{-1,} 975 \mathrm{~cm}^{-1,} 1030 \mathrm{~cm}^{-1,} 1380$ $\mathrm{cm}^{-1}, 1570 \mathrm{~cm}^{-1}$ corresponding to $\mathrm{W}=\mathrm{O}, \mathrm{O}-\mathrm{W}-\mathrm{O}, \mathrm{N}-\mathrm{H}, \mathrm{C}-\mathrm{N}$ and $\mathrm{C}=\mathrm{C}$ groups, respectively with shift due to adsorption procedure (Figure 10).

Table 4 compares the catalytic activity of the supported catalyst with those of other reported catalysts for the oxidation of alkenes.

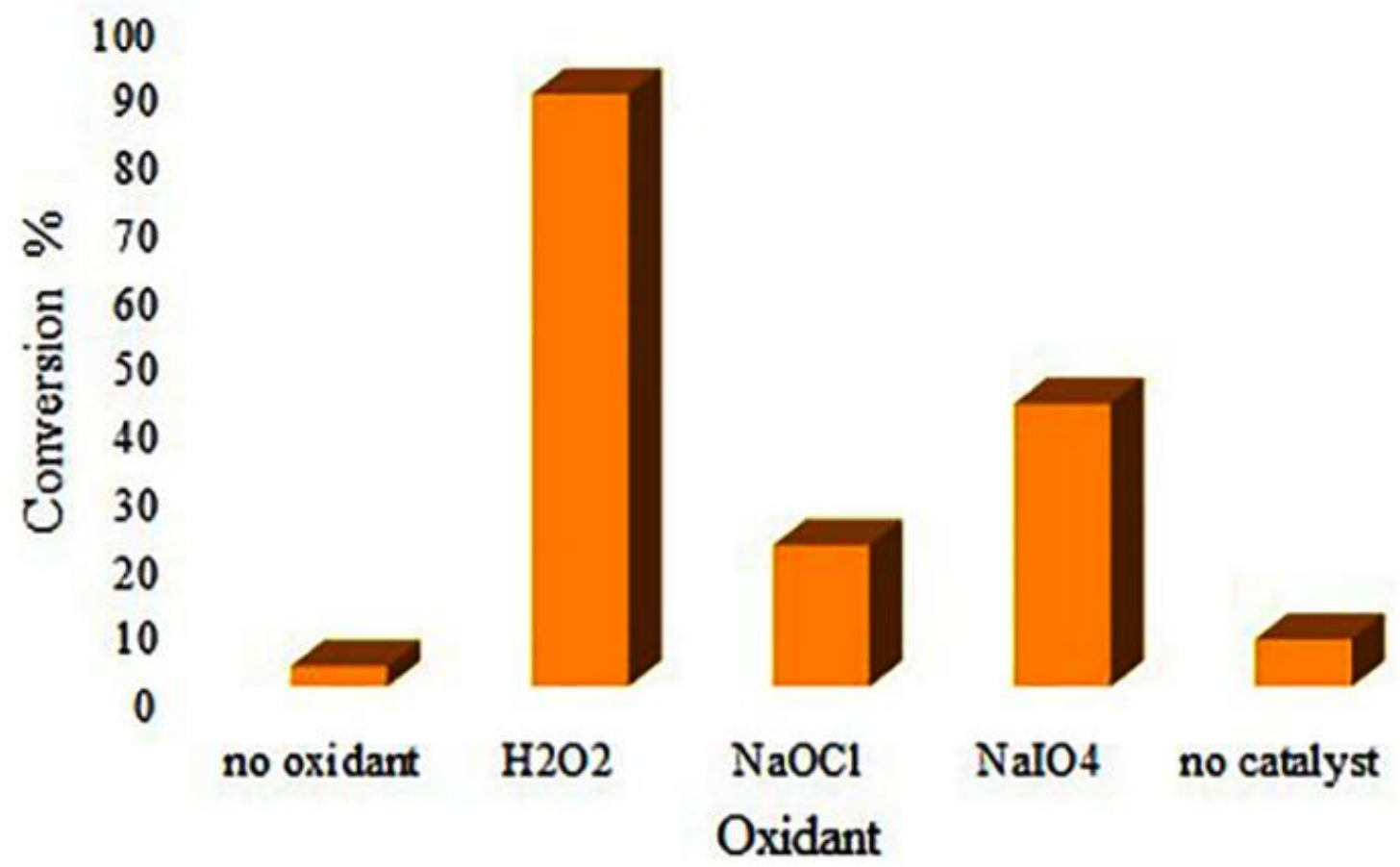

Figure 8. Effect of oxidant nature on oxidation of cyclooctene to epoxide in optimized conditions 


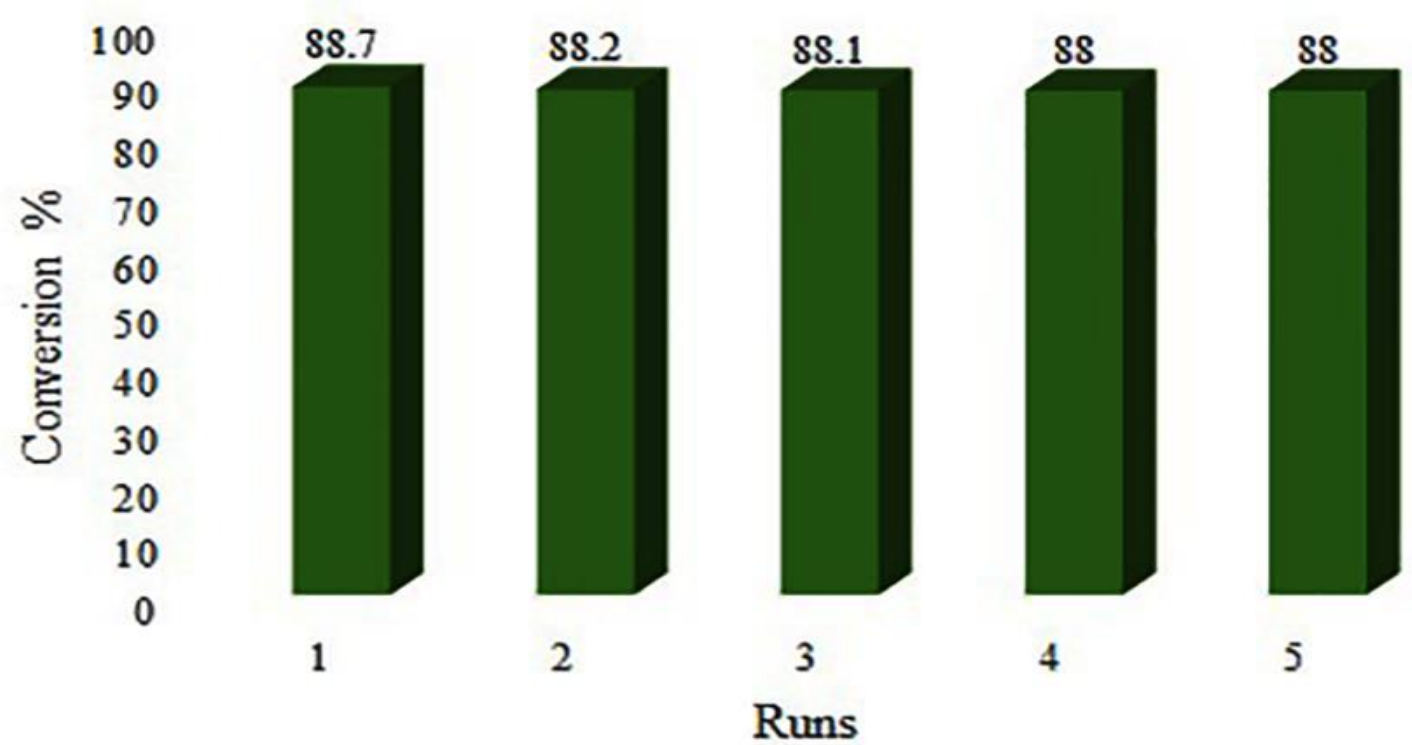

Figure 9. Recycling of the catalytic system for epoxidation of cyclooctene in $\mathrm{THF} / \mathrm{CH}_{3} \mathrm{CN}$ according to the procedure mentioned in Table 4.

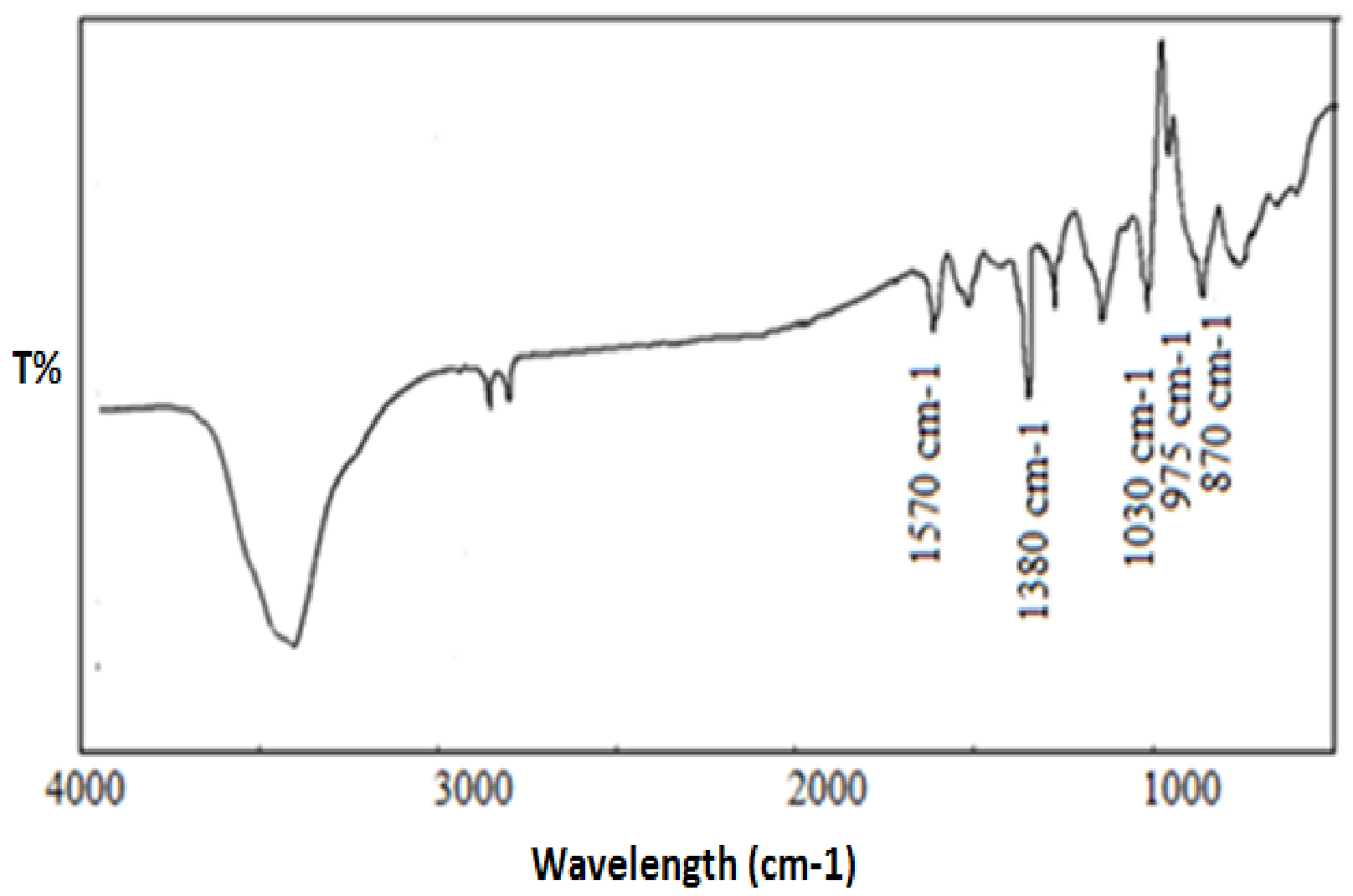

Figure 10. FT-IR spectrum of reused catalyst after oxidation of cycloocten 
Table. 4. Comparison of reaction data for the present method in oxidation of alkenes and other reported methods

\begin{tabular}{ccc} 
Catalyst & Epoxide \% & References \\
HPW@Ppy & Cyclooctene & This work \\
Keggin POM & Cyclooctene & {$[7-9]$} \\
POM@ Polyaniline & Alkenes & {$[10]$} \\
Keggin POM @ Bentonite & Alkenes & {$[11]$} \\
\hline
\end{tabular}

\section{Conclusion}

HPW@Ppy nanocomposite was prapared by anchoring HPW on the surfaces of Ppy. The HPW@Ppy catalyst showed excellent catalytic activity in oxidation procedures and could, therefore, be reused at least five times, with only a slight decrease in photocatalytic activity. In addition, this catalyst could be easily separated at the end of the reaction. The results showed that supported catalyst could accelerate electron transfer in catalytic procedures compared to intact polymer.

\section{Acknowledgments}

The authors are thankful to Payame Noor University in Isfahan Research Council and contributions from Brijand University are gratefully acknowledged.

\section{References}

[1]. Pope M.T., Muller A. Angew. Chem. Int. Edi. Eng., 1991, 30:34

[2]. Tangestaninejad S., Moghadam M., Mirkhani V., Mohammadpoor I., Salavati H. J. Iran. Chem. Soc., 2010, 71:61

[2]. Marchena C.L., Lerici L., Renzini S., Pierella L., Pizzio L. Appl. Catal. B Environ, 2016, 188:23

[3]. Duncan D.C., Netzel T.L., Hill C.L. Inorg. Chem., 1995, 18:4640

[4]. Guo Y., Hu C., Jiang C., Yang Y., Jiang S. X. J. Catal., 217. 2003, 1:141

[5]. Licini G., Conte V., Coletti A., Mba M., Zonta C., Coord Chem. Rev., 2011, 255:2345

[6]. Toufaily J., Soulard M., Delmote L., Colloids Surf A: Physicochem Engin Aspects., 2008, 1:285

[7]. Khenkin A.M., Hill C.L. Commun., 1993, 3:140

[8]. Zhang X., Chen Q., Duncan D.C., Lachicotte R.J., Hill C.L., Inorg. Chem., 1997, 36:4381

[10]. Farsani M.R., Jalilian F., Yadollahi B., Rudbari H.A., Appl. Organometal. Chem., 2015, 29:7

[9]. Salavati H., Rasouli N. Appl. Surf.Sci., 2011, 257:4532 
[11]. Salavati H., Rasouli N. Mat. Res. Bull., 2011, 46:1853

[11]. Hamadi H., Kooti M., Afshari M., Ghiasifar Z. J. Mol. Catal. A: Chem., 2013, 373:25

[12]. Zheng X., Zhang L., Li J., Luo S. Chem. Commun., 2011, 45:12325

[13]. Cabrera L., Gutierrez S., Herrasti P. J. Magnetism Magnetic Mater, 2009, 321:2115

[14]. Bonastre J., Lapuente R., Garcés P., Cases F., Synth. Met., 2009, 159:1723

[15]. Yang L., Enbo W., Yonghai S., Yanli S., Zhenhui K., Lin X., Zhuang L., Polymer, 2006, 47:1480

[16]. Haibin J., Shuliang L., Xiaohong Z. Mol., 2016, 21:833

[17]. Mondal P., Roy K., Bayen S.P., Chowdhury P., Talanta., 2011, 83:1482

[18]. Marc G., Garcı Lopez E., Bellardita M., Parisi F., Colbeau-Justin C. Phys. Chem. Chem. Phys., 2013, 15:13329

[19]. Lippens B.C., Boer J.H.J. Catal, 1965, 4:319

[20]. Smith K., Musson A., Deboos G.A.J. Org. Chem., 1998, 63:8448

[21]. Kwok T.T., Tayasuriya K. J. Org. Chem., 1994, 59:4939

[22]. Tanabe K., Misono M., Ono Y. Surf. Sei. Catal., 1989, 51:17

How to cite this manuscript: Hossein Salavati* , Abbas Teimouri, Shahnaz Kazemi. Synthesis and characterization of nanocomposite based on polymer as a highly effective supported catalyst in oxidation of alkenes. Asian Journal of Green Chemistry, 2017, 1, 98-115. DOI:10.22631/ajgc.2017.94610.1012 